\title{
Diagnostic Accuracy of Centrally Restricted Diffusion in the Differentiation of Treatment-Related Necrosis from Tumor Recurrence in High-Grade Gliomas
}

\author{
(D) N. Zakhari, (D) M.S. Taccone, (D). Torres, DS. Chakraborty, (D). Sinclair, (D). Woulfe, (D) G.H. Jansen, and (D)T.B. Nguyen
}

\begin{abstract}
BACKGROUND AND PURPOSE: Centrally restricted diffusion has been demonstrated in recurrent high-grade gliomas treated with bevacizumab. Our purpose was to assess the accuracy of centrally restricted diffusion in the diagnosis of radiation necrosis in high-grade gliomas not treated with bevacizumab.
\end{abstract}

MATERIALS AND METHODS: In this prospective study, we enrolled patients with high-grade gliomas who developed a new ringenhancing necrotic lesion and who underwent re-resection. The presence of a centrally restricted diffusion within the ring-enhancing lesion was assessed visually on diffusion trace images and by ADC measurements on 3T preoperative diffusion tensor examination. The percentage of tumor recurrence and radiation necrosis in each surgical specimen was defined histopathologically. The association between centrally restricted diffusion and radiation necrosis was assessed using the Fisher exact test. Differences in ADC and the ADC ratio between the groups were assessed via the Mann-Whitney $U$ test, and receiver operating characteristic curve analysis was performed.

RESULTS: Seventeen patients had re-resected ring-enhancing lesions: 8 cases of radiation necrosis and 9 cases of tumor recurrence. There was significant association between centrally restricted diffusion by visual assessment and radiation necrosis $(P=.015)$ with a sensitivity of $75 \%$ and a specificity of $88.9 \%$, a positive predictive value $85.7 \%$, and a negative predictive value of $80 \%$ for the diagnosis of radiation necrosis. There was a statistically significant difference in the ADC and ADC ratio between radiation necrosis and tumor recurrence $(P=.027)$.

CONCLUSIONS: The presence of centrally restricted diffusion in a new ring-enhancing lesion might indicate radiation necrosis rather than tumor recurrence in high-grade gliomas previously treated with standard chemoradiation without bevacizumab.

ABBREVIATIONS: $\mathrm{HGG}=$ high-grade glioma; $\mathrm{RN}=$ radiation necrosis

$\mathbf{T}$ he appearance of a new ring-enhancing lesion after surgery and chemoradiation for high-grade glioma (HGG) can be seen in tumor recurrence and radiation necrosis $(\mathrm{RN})$. A radiation-induced ring-enhancing lesion contains coagulative necrosis secondary to vascular endothelial injury and fibrinoid necrosis of small blood vessels. ${ }^{1}$ On the other hand, the nonenhancing necrotic com-

Received April 20, 2017; accepted after revision October 17.

From the Department of Radiology (N.Z., C.T., S.C., T.B.N.), Division of Neuroradiology, University of Ottawa, The Ottawa Hospital Civic and General Campus, Ottawa, Ontario, Canada; and Division of Neurosurgery (M.S.T., J.S.) and Department of Pathology and Laboratory Medicine (J.W., G.H.J.), University of Ottawa, The Ottawa Hospital Civic Campus, Ottawa, Ontario, Canada.

This work was supported by the Brain Tumour Foundation of Canada (grant awarded in 2012)

Paper previously presented at: Annual Meeting of the American Society of Neuroradiology and the Foundation of the ASNR Symposium, April 22-27, 2017; Long Beach, California.

Please address correspondence to Thanh B. Nguyen, MD, FRCPC, Department of Radiology, Division of Neuroradiology, University of Ottawa, The Ottawa Hospital Civic and General Campus, 1053 Carling Ave, Ottawa, Ontario, Canada, K1Y 4E9;

e-mail: thnguyen@toh.ca

http://dx.doi.org/10.3174/ajnr.A5485 ponent in recurrent glioblastoma contains liquefactive necrosis. ${ }^{2}$ Differentiation between the 2 remains difficult on conventional MR imaging. ${ }^{3-6}$ Diffusion restriction has been reported in HGGs treated with bevacizumab ${ }^{2}$ and in radiation necrosis of metastatic brain lesions. ${ }^{7}$ Our purpose was to assess the accuracy of centrally restricted diffusion in the diagnosis of RN in HGG not treated with bevacizumab.

\section{MATERIALS AND METHODS Patient Population}

We consecutively enrolled patients with HGG who developed a new enhancing lesion on follow-up MR imaging after resection, radiation (60 Gy in 30 fractions of 2 Gy during 6 weeks), and temozolomide therapy, from December 2012 to December 2016. The patients were part of a prospective diagnostic accuracy study on dynamic contrastenhanced and DSC MR imaging for glioma recurrence, approved by our institutional research ethics board. Written informed consent was obtained from all the patients. For the assessment of central diffusion restriction in newly developed necrotic enhancing lesions, the inclusion criteria were the presence of a central area of necrosis 
within the newly enhancing lesion and surgical re-resection of this lesion. The exclusion criteria were an entirely solid newly enhancing lesion, bevacizumab therapy, and an operation $>3$ months after the study MR imaging. A short interval between the operation and the MR imaging was chosen to ensure that the pathology results were representative of the lesions visualized on the imaging study.

\section{MR Imaging Acquisition Protocols}

Each patient was scanned on a 3T MR imaging scanner (Magnetom Trio; Siemens, Erlangen, Germany) with the following parameters: conventional MR imaging protocol using axial $\mathrm{T} 1$ precontrast $\left(\mathrm{TR}=280 \mathrm{~ms}, \mathrm{TE}=2.51 \mathrm{~ms}\right.$, flip angle $=90^{\circ}$, voxel size $=1.1 \times 0.9 \times 3 \mathrm{~mm})$, axial FLAIR $(\mathrm{TR}=9710 \mathrm{~ms}$, $\mathrm{TE}=93 \mathrm{~ms}, \mathrm{TI}=2580 \mathrm{~ms}$, voxel size $=1.1 \times 0.9 \times 3 \mathrm{~mm})$, axial $\mathrm{T} 2(\mathrm{TR}=6910 \mathrm{~ms}, \mathrm{TE}=97 \mathrm{~ms}$, voxel size $=0.7 \times 0.7 \times$ $3 \mathrm{~mm}$ ), and a postcontrast axial T1 volumetric interpolated brain examination $(\mathrm{TR}=8.48 \mathrm{~ms}, \mathrm{TE}=3.21 \mathrm{~ms}$, flip angle $=$ $12^{\circ}$, voxel size $\left.=1 \times 1 \times 1 \mathrm{~mm}\right)$. We used an IV injection of 0.1 $\mathrm{mmol} / \mathrm{kg}$ of gadobutrol (Gadovist 1.0; Bayer Schering Pharma, Berlin, Germany). Diffusion tensor imaging was performed with the following parameters: $\mathrm{TR}=3100 \mathrm{~ms}, \mathrm{TE}=106 \mathrm{~ms}, 20$ directions, $b=0 / 1000$, voxel size $=1.3 \times 1.3 \times 2.5 \mathrm{~mm}$.

\section{MR Image Interpretation and Data Collection}

The ADC maps were coregistered to the axial T1-weighted postcontrast images using a commercially available software (Olea Sphere 1.3; Olea Medical, La Ciotat, France). Qualitative and quantitative assessments were performed by 1 neuroradiologist (N.Z.) blinded to the clinical information and histopathologic results. The presence of centrally restricted diffusion within part or all of the necrotic component was visually assessed in each ring-enhancing lesion and recorded as present or absent. Central diffusion restriction was defined as higher signal on trace images with lower signal on ADC compared with the rest of the lesion. For quantitative analysis, ADC values were calculated from DTI. Separate ROIs (Fig 1C) were manually placed within the necrotic component of each lesion excluding the enhancing wall, as well as within the enhancing component of the lesions, and the minimum ADC was recorded. The ROI size was kept between 20 and $30 \mathrm{~mm}^{2}$. ROIs were placed in the contralateral normal-appearing white matter. We calculated the following ADC ratios: ADC Necrosis Ratio = ADC Central Necrosis/ADC White Matter; ADC Enhancement Ratio = ADC Enhancing Component/ADC White Matter; and ADC Necrosis/Enhancement Ratio = ADC Central Necrosis/ADC Enhancing Component.
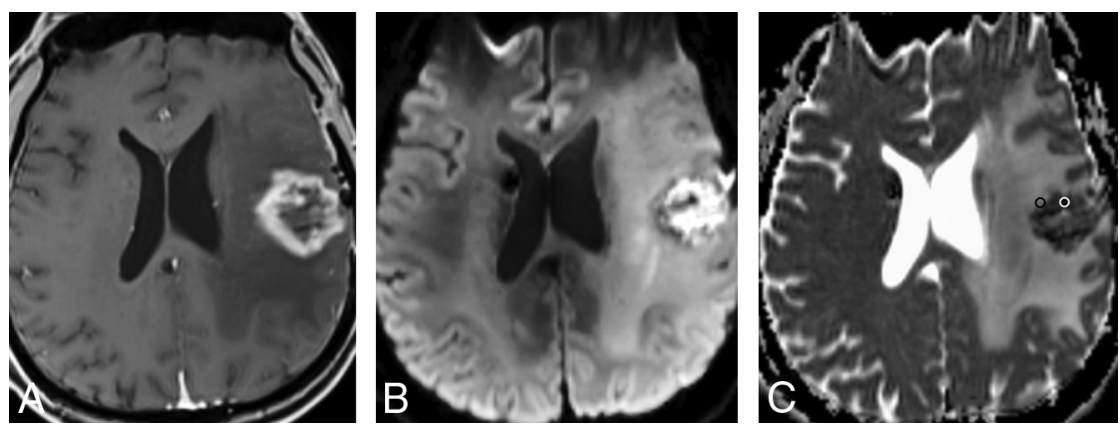

FIG 1. Radiation necrosis: $A$, Axial T1-weighted MR image shows a left frontal necrotic ringenhancing lesion. $B$ and $C$, Axial DWI and ADC map demonstrate diffusion restriction within the necrotic center of the lesion. Representative ROIs are placed on the ADC map corresponding to the enhancing rim (black ROI) and the necrotic component (white ROI).

\section{Histopathologic Analysis}

Following surgical resection, an experienced neuropathologist (J.W. or G.H.J.), blinded to the imaging findings, determined the presence or absence of viable tumor and/or radiation necrosis using the 2007 World Health Organization classification and estimated the percentage of viable tumor and radiation necrosis within the specimen. Visual assessment was performed under $\times 4$ high-power-field microscopy to determine the approximate percentage area of radiation necrosis or tumor recurrence relative to the whole tissue area. The following criteria were used
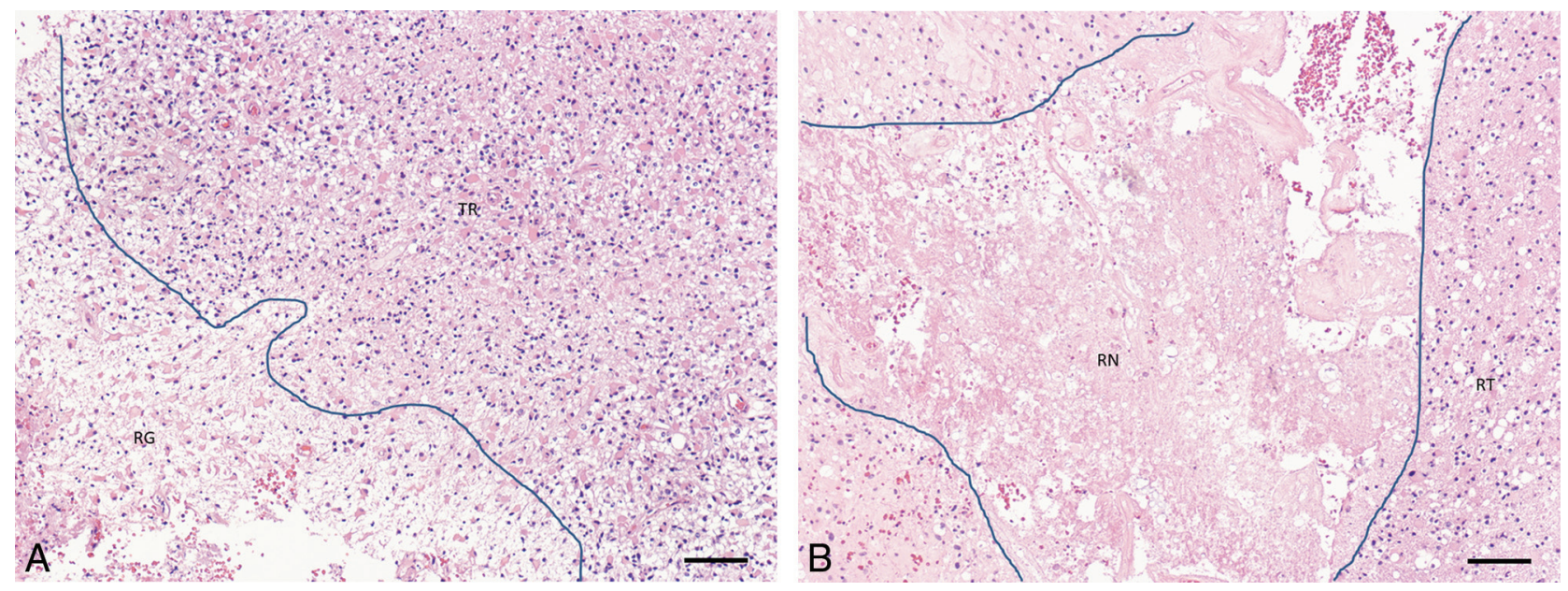

FIG 2. $A$, Specimen showing recurrent tumor (TR) adjacent to an area of brain tissue showing reactive gliosis (RG). $B$, Specimen showing predominantly radiation necrosis (RN, center) with a small amount of viable tumor peripherally containing residual glioma (RT). Lines in both images show interface between reactive brain and radiation necrosis, respectively, with recurrent tumor. Both sections stained with hematoxylin and eosin. Scale bars $=100$ microns. 
for the diagnosis of radiation necrosis: the presence of coagulative necrosis, hyalinized vessels, and microcalcifications. For the diagnosis of recurrent tumor, we used the following criteria: the presence of increased cellularity and nuclear pleomorphism. Given that most lesions consisted of a mixture of tumor- and treatment-related changes in different proportions, a lesion was categorized as predominantly tumor recurrence or predominantly $\mathrm{RN}$ based on the component with the higher proportion in the specimen (Fig 2).

\section{Statistical Analysis}

The association between the presence of centrally restricted diffusion and pathologically proved radiation necrosis was assessed

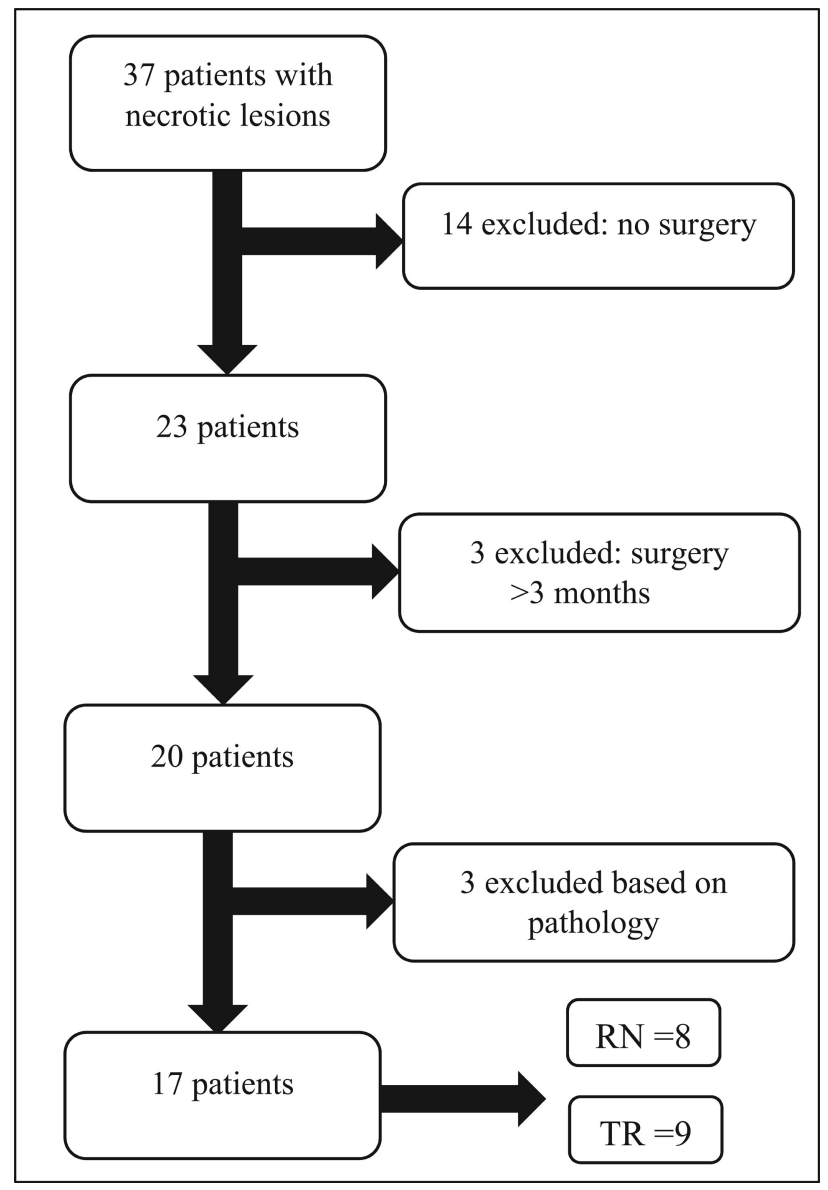

FIG 3. Flow chart illustrating patient selection. TR indicates tumor recurrence.
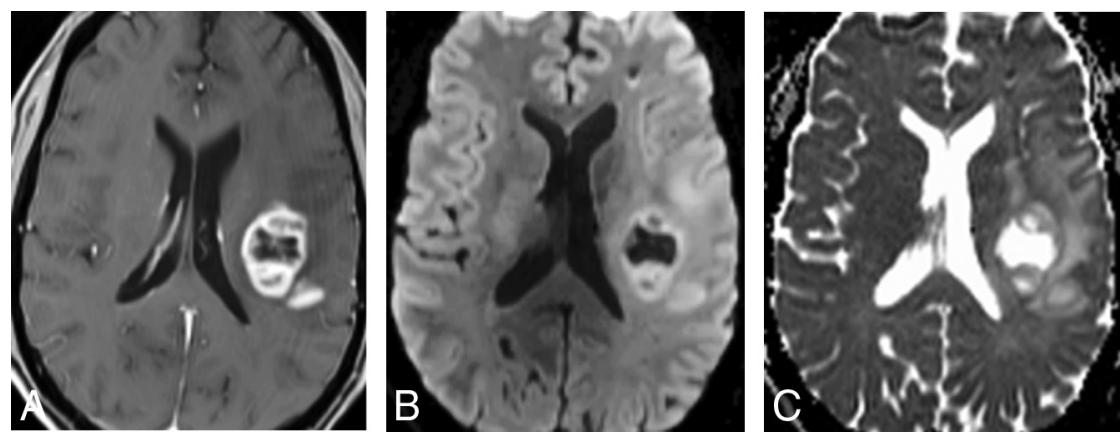

FIG 4. Tumor recurrence. $A$, Axial T1-weighted MR image shows a left frontal necrotic ringenhancing lesion. $B$ and $C$, Axial DWI and ADC map demonstrate facilitated diffusion within the necrotic center of the lesion. using the Fisher exact test, and the diagnostic accuracy of this finding was calculated using receiver operating characteristic curve analysis. Differences in the minimum ADC and ADC ratio of the necrotic component as well as the enhancing rim between radiation necrosis and tumor recurrence were assessed via the Mann-Whitney $U$ test. A $P$ value of .05 indicated statistical significance. All data were analyzed with MedCalc for Windows (Version 12; MedCalc Software, Mariakerke, Belgium).

\section{RESULTS}

Thirty-seven patients with HGG with new necrotic peripherally enhancing lesions were identified. Twenty-three patients had reresection of the identified lesions, and 14 had nonsurgical management. Three patients had an operation $>3$ months after the study MR imaging. Three patients were subsequently excluded on the basis of the pathology results: Two had equal proportions of necrosis and tumor, and 1 had hemorrhage and pus with no evidence of tumor recurrence or RN (Fig 3). Of note, the lesion excluded for presence of pus and blood product in the center of the lesion at the operation had central diffusion restriction on imaging.

There were 13 men and 4 women in the included patients. Average age was $55.9 \pm 10.3$ years (range, $33.3-77.6$ years). The average duration between the operation and the MR imaging examination was $25.8 \pm 23.99$ days (range, $1-78$ days).

\section{Qualitative Assessment}

Nine patients had predominant tumor recurrence, and 8 had predominant RN (Fig 3). The presence of centrally restricted diffusion in the necrotic lesions was significantly associated with the predominance of $\mathrm{RN}$ on histopathologic analysis $(P=.015)$ (Figs 1 and 4 and Table 1). Central diffusion restriction was seen in 6 patients with RN. From the 9 patients with predominant tumor recurrence, only 1 patient had central diffusion restriction. On histopathologic analysis, radiation necrosis comprised approximately $30 \%$ of this patient's surgical specimen. The presence of central diffusion restriction had a sensitivity of $75 \%$ and a specificity of $88.9 \%$, with a positive predictive value of $85.7 \%$ and a negative predictive value of $80 \%$ for the diagnosis of radiation necrosis in a newly developed necrotic lesion after treatment of HGG. From the 20 patients who were excluded, 9 patients had central diffusion restriction on visual inspection.

\section{Quantitative Assessment}

There was a significant difference in the minimum ADC and ADC ratio in the necrotic component of the tumor recurrence and $\mathrm{RN}$ lesions $(P=$ .027) with a lower median ADC and $\mathrm{ADC}$ ratio in the $\mathrm{RN}$ group compared with the tumor recurrence group (Table 2). On the basis of the receiver operating characteristic curve analysis (area under the curve $=0.82 ; 95 \% \mathrm{CI}, 0.5-0.96 ; P=$ .002 ), an ADC cutoff value of $\leq 1316 \times$ $10^{-6} \mathrm{~mm}^{2} / \mathrm{s}$ provides $100 \%$ specificity for RN but only $55.6 \%$ sensitivity. Using a cutoff of $\leq 1197.03 \times 10^{-6} \mathrm{~mm}^{2} / \mathrm{s}$ results in $80 \%$ specificity and $66.7 \%$ sensitivity for the identification of RN. Similarly, an ADC necrosis ratio cutoff value 
of $\leq 1.78$ provides $100 \%$ specificity and $55.6 \%$ sensitivity for the identification of RN (Fig 5).

No significant difference was found in the ADC and ADC ratio of the enhancing components between tumor recurrence and $\mathrm{RN}(P=$ .63 and $P=.85)$ (Table 2). However, there was a significant difference in the necrotic-to-enhancing component $\operatorname{ADC}$ ratio $(P=.043)$, with a lower ratio in the RN group (Table 2). On the basis of the receiver operating characteristic curve analysis (area under the curve $=0.79 ; 95 \%$ CI, $0.53-0.95 ; P=.001$ ), a cutoff value of $\leq 0.95$ provides $88.9 \%$ sensitivity and $62.5 \%$ specificity for RN (Fig 5).

\section{DISCUSSION}

The results of the current study suggest that evaluation of diffusion restriction in the nonenhancing necrotic center rather than the peripheral enhancing component of ring-enhancing lesions developing after treatment of HGG may be a useful tool in differentiating RN from tumor recurrence. Central diffusion restriction is associated with a predominance of radiation necrosis on pathology, with a lower minimum ADC in the lesion center in $\mathrm{RN}$ compared with tumor recurrence.

Most of the previously published studies assessing the diagnostic accuracy of DWI in differentiating tumor recurrence and $\mathrm{RN}$ focused on assessment of diffusion restriction and ADC values in the enhancing component of the lesion with the expectation that the increased cellularity in tumor recurrence and increased extracellular space associated with cell death in RN would result in diffusion restriction and lower ADC values with tumor recurrence. ${ }^{8-10}$ However, the results were not consistent. ${ }^{11-15} \mathrm{~A}$ meta-analysis of the diagnostic accuracy of DWI showed moderate diagnostic performance and recommended against the use of DWI alone in differentiating $\mathrm{RN}$ and tumor recurrence. ${ }^{16}$

Like the results of the current study, evaluation of the central zone of radiation necrosis and malignant glioma in rat models

Table 1: Results of qualitative assessment for the presence of centrally restricted diffusion

\begin{tabular}{lcc}
\hline & \multicolumn{3}{c}{$\begin{array}{c}\text { Central Diffusion } \\
\text { Restriction }\end{array}$} \\
\cline { 2 - 3 } Diagnosis & Present & Absent \\
\hline RN & 6 & 2 \\
TR & 1 & 8 \\
\hline
\end{tabular}

Note:-TR indicates tumor recurrence. identified a pattern of central diffusion restriction in radiation necrosis with significantly lower ADC values compared with malignant glioma. The diffusion restriction in the central zone of radiation necrosis corresponded to coagulative necrosis on histopathology. ${ }^{12}$

Asao et $\mathrm{al}^{17}$ reported that most of the radiation necrosis lesions in their patient population, consisting predominantly of astrocytic neoplasms, had heterogeneous signal intensity on DWI with hyperintensities within the lesions. There are also a few reported cases in the literature with diffusion restriction in the center of radiation necrosis, simulating central diffusion restriction in the abscess. ${ }^{18,19}$ In fact, one of the cases that showed central diffusion restriction in our series was found to have infection and hemorrhage within the lesion center at the operation.

Nonenhancing diffusion restriction has also been reported in bevacizumab-treated $\mathrm{HGG}^{20,21}$ as well as bevacizumab-treated brain metastasis. ${ }^{22}$ Nguyen et $\mathrm{al}^{2}$ demonstrated that progressively expanding diffusion restriction in HGG treated with bevacizumab pathologically represents coagulative necrosis surrounded by viable tumor cells. They also showed lower ADC in the diffusion-restricted necrosis compared with the hypercellular nonenhancing tumor.

In their series of treated metastatic lesions, Cha et $\mathrm{al}^{7}$ described a 3-layer pattern of radiation necrosis with a central core of lique-

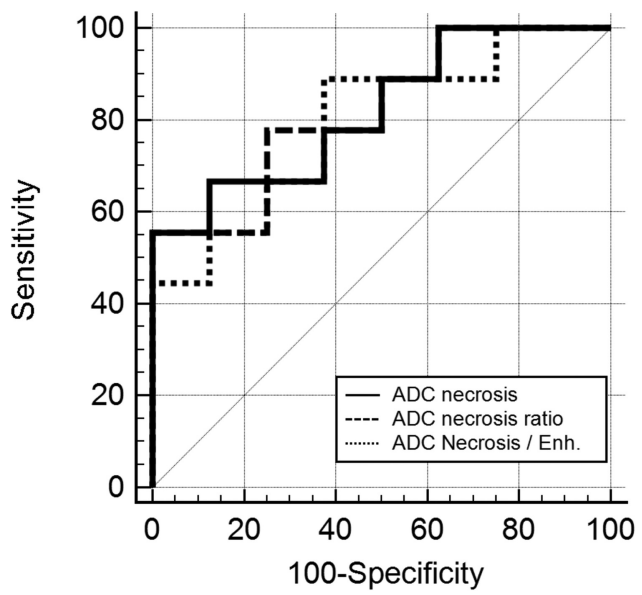

FIG 5. Graph displaying ADC central necrosis, the ADC necrosis ratio, and $A D C$ necrosis/enhancement receiver operating characteristic curves.

Table 2: Median ADC values and ADC ratios of the necrotic and enhancing components for radiation necrosis and tumor recurrence ${ }^{\mathrm{a}}$

\begin{tabular}{|c|c|c|c|c|c|}
\hline \multirow[b]{2}{*}{ ADC } & \multicolumn{2}{|c|}{ RN } & \multicolumn{2}{|c|}{ TR } & \multirow[b]{2}{*}{$P$} \\
\hline & Median & $95 \% \mathrm{Cl}$ & Median & $95 \% \mathrm{Cl}$ & \\
\hline Necrosis $\left(10^{-6} \mathrm{~mm}^{2} / \mathrm{s}\right)$ & $\begin{array}{c}992.15 \\
(755.06-1197.29)\end{array}$ & $684.72-1220.80$ & $\begin{array}{c}1759.86 \\
(1136.70-2401.44)\end{array}$ & 1054.80-2588.01 & .027 \\
\hline Necrosis ratiob & $\begin{array}{c}1.24 \\
(0.96-1.57)\end{array}$ & $0.91-1.74$ & $2.21(1.41-2.9)$ & $1.31-3.32$ & .027 \\
\hline Enhancement $\left(10^{-6} \mathrm{~mm}^{2} / \mathrm{s}\right)$ & $\begin{array}{c}1044.37 \\
(945.09-1229.65)\end{array}$ & $867.08-1245.53$ & $\begin{array}{c}1106.70 \\
(958.53-1200.02)\end{array}$ & $955.86-1241.24$ & .63 \\
\hline Enhancement ratio $^{c}$ & $\begin{array}{c}1.38 \\
(1.13-1.55)\end{array}$ & $1.02-1.59$ & 1.39 (1.14-1.51) & $1.12-1.60$ & .85 \\
\hline Necrosis/enhancement ${ }^{d}$ & $\begin{array}{c}0.93 \\
(0.74-1.07)\end{array}$ & $0.56-1.27$ & $1.59(0.98-2.3)$ & $0.96-2.41$ & .043 \\
\hline
\end{tabular}

Note:-TR indicates tumor recurrence.

${ }^{a}$ Interquartile ranges are in parentheses.

${ }^{b}$ Necrosis Ratio = ADC Central Necrosis/ADC White Matter.

${ }^{c}$ Enhancement Ratio = ADC Enhancing Component/ADC White Matter.

${ }^{\mathrm{d}}$ Necrosis/Enhancement $=$ ADC Central Necrosis/ADC Enhancing Component. 
fied necrosis showing increased ADC, a nonenhancing middle layer of coagulative necrosis with low ADC, and an outer layer of elevated CBV representing a zone of active inflammation. Our findings of diffusion restriction centrally in the nonenhancing component along the inner aspect of the enhancing ring likely correspond to the middle layer in their 3-layer pattern. They also showed lower minimum ADC values in RN compared with tumor recurrence, comparable with the current study. ${ }^{7}$

In this study, we included only surgically proved cases, leading to the small number of patients in this series, which is the main limitation to the study. Although only surgically proved cases were included, the pathologic samples were not limited to the areas of diffusion restriction, another limitation to this study. While we classified the lesions into predominant tumor recurrence or RN on the basis of the component with the higher proportion in the surgical specimen, the proportion of tumor in the cases classified as predominant $\mathrm{RN}$ in this series ranged from $5 \%$ to $20 \%$. However, it can still be argued from a pathologic point of view that the presence of any amount of tumor cells in the specimen should be considered evidence of tumor recurrence. Future studies with image-guided stereotactic biopsy of the regions of centrally restricted diffusion would be of great value in confirming the current findings. The results of this study cannot be generalized to patients treated with bevacizumab or lesions with no necrosis.

\section{CONCLUSIONS}

This study shows that the presence of central nonenhancing restricted diffusion in a newly necrotic ring-enhancing lesion might indicate RN rather than tumor recurrence in HGG previously treated with standard chemotherapy without bevacizumab.

Disclosures: Santanu Chakraborty-UNRELATED: Grants/Grants Pending: Bayer Healthcare Pharmaceuticals, GE Healthcare. John Woulfe-UNRELATED: Grants/ Grants Pending: Parkinson Society Canada, Canadian Institutes of Health Research. Thanh B. Nguyen—RELATED: Grant: Brain Tumour Foundation of Canada*; UNRELATED: Grants/Grants Pending: Bayer Healthcare Pharmaceuticals, GE Healthcare, Radiological Society of North America. *Money paid to the institution.

\section{REFERENCES}

1. Chao ST, Ahluwalia MS, Barnett GH, et al. Challenges with the diagnosis and treatment of cerebral radiation necrosis. Int J Radiat Oncol Biol Phys 2013;87:449-57 CrossRef Medline

2. Nguyen HS, Milbach N, Hurrell SL, et al. Progressing bevacizumabinduced diffusion restriction is associated with coagulative necrosis surrounded by viable tumor and decreased overall survival in patients with recurrent glioblastoma. AJNR Am J Neuroradiol 2016; 37:2201-08 CrossRef Medline

3. Kumar AJ, Leeds NE, Fuller GN, et al. Malignant gliomas: MR imaging spectrum of radiation therapy- and chemotherapy-induced necrosis of the brain after treatment. Radiology 2000;217:377-84 CrossRef Medline

4. Mullins ME, Barest GD, Schaefer PW, et al. Radiation necrosis versus glioma recurrence: conventional MR imaging clues to diagnosis. AJNR Am J Neuroradiol 2005;26:1967-72 Medline

5. Hygino da Cruz LC Jr, Rodriguez I, Domingues RC, et al. Pseudoprogression and pseudoresponse: imaging challenges in the assessment of posttreatment glioma. AJNR Am J Neuroradiol 2011;32: 1978-85 CrossRef Medline

6. Fatterpekar GM, Galheigo D, Narayana A, et al. Treatment-related change versus tumor recurrence in high-grade gliomas: a diagnostic conundrum - use of dynamic susceptibility contrast-enhanced (DSC) perfusion MRI. AJR Am J Roentgenol 2012;198:19-26 CrossRef Medline

7. Cha J, Kim ST, Kim HJ, et al. Analysis of the layering pattern of the apparent diffusion coefficient (ADC) for differentiation of radiation necrosis from tumour progression. Eur Radiol 2013;23:879-86 CrossRef Medline

8. Shah R, Vattoth S, Jacob R, et al. Radiation necrosis in the brain: imaging features and differentiation from tumor recurrence. $R a$ diographics 2012;32:1343-59 CrossRef Medline

9. Chan YL, Yeung DK, Leung SF, et al. Diffusion-weighted magnetic resonance imaging in radiation-induced cerebral necrosis: apparent diffusion coefficient in lesion components. J Comput Assist Tomogr 2003;27:674-80 CrossRef Medline

10. Hein PA, Eskey CJ, Dunn JF, et al. Diffusion-weighted imaging in the follow-up of treated high-grade gliomas: tumor recurrence versus radiation injury. AJNR Am J Neuroradiol 2004;25:201-09 Medline

11. Rock JP, Scarpace L, Hearshen D, et al. Associations among magnetic resonance spectroscopy, apparent diffusion coefficients, and image-guided histopathology with special attention to radiation necrosis. Neurosurgery 2004;54:1111-17, discussion 1117-19 Medline

12. Wang S, Chen Y, Lal B, et al. Evaluation of radiation necrosis and malignant glioma in rat models using diffusion tensor MR imaging. J Neurooncol 2012;107:51-60 CrossRef Medline

13. Kazda T, Bulik M, Pospisil P, et al. Advanced MRI increases the diagnostic accuracy of recurrent glioblastoma: single institution thresholds and validation of MR spectroscopy and diffusion weighted MR imaging. Neuroimage Clin 2016;11:316-21 CrossRef Medline

14. Lee WJ, Choi SH, Park CK, et al. Diffusion-weighted MR imaging for the differentiation of true progression from pseudoprogression following concomitant radiotherapy with temozolomide in patients with newly diagnosed high-grade gliomas. Acad Radiol 2012;19: 1353-61 CrossRef Medline

15. Song YS, Choi SH, Park CK, et al. True progression versus pseudoprogression in the treatment of glioblastomas: a comparison study of normalized cerebral blood volume and apparent diffusion coefficient by histogram analysis. Korean J Radiol 2013;14:662-72 CrossRef Medline

16. Zhang H, Ma L, Shu C, et al. Diagnostic accuracy of diffusion MRI with quantitative ADC measurements in differentiating glioma recurrence from radiation necrosis. J Neurol Sci 2015;351:65-71 CrossRef Medline

17. Asao C, Korogi Y, Kitajima M, et al. Diffusion-weighted imaging of radiation-induced brain injury for differentiation from tumor recurrence. AJNR Am J Neuroradiol 2005;26:1455-60 Medline

18. Tung GA, Evangelista P, Rogg JM, et al. Diffusion-weighted MR imaging of rim-enhancing brain masses: is markedly decreased water diffusion specific for brain abscess? AJR Am J Roentgenol 2001;177: 709-12 CrossRef Medline

19. Biousse V, Newman NJ, Hunter SB, et al. Diffusion weighted imaging in radiation necrosis. J Neurol Neurosurg Psychiatry 2003;74: 382-84 CrossRef Medline

20. Farid N, Almeida-Freitas DB, White NS, et al. Restriction-spectrum imaging of bevacizumab-related necrosis in a patient with GBM. Front Oncol 2013;3:258 CrossRef Medline

21. Rieger J, Bähr O, Müller K, et al. Bevacizumab-induced diffusionrestricted lesions in malignant glioma patients. J Neurooncol 2010; 99:49-56 CrossRef Medline

22. Sivasundaram L, Hazany S, Wagle N, et al. Diffusion restriction in a non-enhancing metastatic brain tumor treated with bevacizumab: recurrent tumor or atypical necrosis? Clin Imaging 2014;38:724-26 CrossRef Medline 